\title{
Las habilidades directivas clave para el desarrollo organizacional
}

\section{González Marin, Yesid ${ }^{\mathrm{a}}$, Canós-Darós, Lourdes ${ }^{\mathrm{b}}$}

${ }^{\mathrm{a}}$ Facultad de Ciencias Economicas Administrativas y Contables, Corporacion Universitaria U de Colombia, Medellin (Colombia), yesidadmon@gmail.com, bepartamento de Organización de Empresas, Escuela Politécnica Superior de Gandia, Universitat Politècnica de València, Valencia (España), loucada@omp.upv.es.

\begin{abstract}
Resumen
Los directivos en las organizaciones son conscientes que el desarrollo del capital humano es un factor clave para el desarrollo de las actividades empresariales con éxito, y supone una orientación consciente, pragmática y dirigida a los procesos que llevarán a fortalecer las habilidades necesarias que debe desarrollar el trabajador en su puesto de trabajo. No obstante, deacuerdo con Junco y Dos-Santos (2017), este nivel de conciencia no está presente en las organizaciones; en concreto en las PYME los directivos no tienen en cuenta el uso de habilidades directivas que permitan abordar responsabilidades de gestión y tomar decisiones eficaces y eficientes.

En este contexto nos preguntamos qué habilidades son la clave del éxito organizacional, basado en la asunción de responsabilidades y la toma de decisiones eficaz y eficiente anteriormente mencionadas. Para encontrar una respuesta, en este trabajo realizamos una revisión de la literatura compilando el conocimiento existente sobre habilidades directivas, determinando su definición, diferentes clasificaciones, e identificando las habilidades más estudiadas por los autores especializados en este tema.
\end{abstract}

Palabras clave: Cultura organizacional, Desempeño organizacional, Gerencia, Gestión, Habilidades directivas.

\section{Introducción}

En el marco de la globalización en donde se desenvuelven las organizaciones, cada vez se hace más relevante que estas se destaquen tanto en procesos internos como externos con objeto de sobrevivir a un mercado de alta competitividad (Zat'ková y Poláček, 2015). Por ello, la capacitación y preparación se ha convertido en una necesidad para que los equipos 
de trabajo cuenten con las herramientas suficientes para enfrentar las problemáticas de la actualidad (Giudici y Filimonau, 2019). En este sentido, las habilidades directivas se circunscriben como pilares fundamentales que direccionan asertivamente las organizaciones hacia el éxito. Sin importar el tipo de organización o tamaño se ha visibilizado la relevancia de fortalecer en este aspecto ya que se convirtieron en una suerte de fórmula trascendental para generar ventaja competitiva en las organizaciones (Bel, Smirnov y Wait, 2018).

El dinamismo interno que se presenta en las organizaciones se relaciona con los roles y especificidades que ejercen sus miembros, no obstante, para lograr potenciar las capacidades de los actores empresariales es necesario dimensionar el escenario de una manera holística y comprender que las organizaciones son un reflejo de la realidad social. En este sentido, los actores empresariales requieren de procesos de aprendizaje, sistemas simbólicos, formas de comunicación, acompañamiento, etc., en suma, aquellos aspectos relacionados con la cultura organizacional (Zapata y Rodríguez, 2017).

El presente trabajo se sirve metodológicamente de una revisión documental, en donde sistemáticamente se realiza la consulta (Toro y Parra, 2010) de aquellas investigaciones relevantes en el tema de las habilidades directivas teniendo en cuenta su definición, clasificación, y ejecución en diferentes escenarios empresariales. De esta manera, se presentan los resultados en tres categorías de análisis. La pertinencia de la revisión se justifica ante la dimensión cada vez más creciente de los aspectos humanos al interior de las organizaciones, que como menciona Codina (2016), se viene planteando desde los años ochenta con el objeto de perfeccionar las técnicas de dirección y gestión en el campo empresarial, además de la necesidad de establecer diversos elementos que median en las relaciones internas y externas que ofrecen no sólo ventajas competitivas sino la mejora del ambiente laboral. Por último, se concibe esta investigación como una contribución a la compilación de las habilidades más significativas que pueden integrarse dentro de los planes estratégicos de los emprendimientos y la toma de decisiones de las empresas que se circunscriben en el panorama actual (Chen et al., 2020).

\section{Definición de habilidades directivas}

Las habilidades directivas son aquellas que permiten un desempeño excepcional y potencian las condiciones del gerente, el cual, contará con la capacidad de tomar decisiones asertivas que convengan a la organización (Tomastika, Strohmandlb y Cechc 2015). Asimismo, hacen alusión al comportamiento para la obtención de resultados, el gerente se sitúa como un líder que toma en cuenta las necesidades de su equipo de trabajo, y los 
resultados para incrementar la eficiencia organizacional y la productividad. De acuerdo con Ali, Zwetsloot y Nada (2019), las habilidades directivas permiten que el gerente asimile y reconfigure tanto los recursos como las competencias de las organizaciones, y a este respecto, enuncian tres dimensiones. La primera es el estilo de gestión donde se dimensiona la influencia que se debe realizar en los colaboradores con el propósito de alcanzar los objetivos organizacionales; el estilo de gestión positiva motiva a los colaboradores a ir más allá de los intereses personales, les permite visibilizar un panorama más amplio (Puchol et al., 2010) La segunda, hace referencia a la toma de decisiones y al proceso mediante el cual las empresas pueden lograr ventajas competitivas en tanto se analicen los indicadores de desempeño, las tendencias del mercado y los aspectos administrativos. Por último, encontramos el desarrollo de las personas, que consiste en proveer de herramientas al equipo de trabajo para la obtención de mayores rendimientos $\mathrm{y}$, por ende, mejores resultados.

En el escenario empresarial actual, las organizaciones se ven en la tarea de concebirse con una superioridad sostenida, esto les permite la supervivencia en dicho entorno. Para ello se requiere de capacidades para identificar, desplegar y preservar recursos que los diferencien de la competencia, por esto las habilidades directivas deben converger en la capacidad de comprensión del escenario cultural junto con la comprensión del escenario organizacional (Varela, Burke y Michel, 2011). De ahí la importancia de conceptualizar las habilidades directivas como un cúmulo de capacidades que dan respuesta a necesidades tanto internas como externas. Asimismo, está la complejidad para establecer una definición estandarizada en tanto que, a pesar del escenario global donde se sitúan las organizaciones en la actualidad, existe un diálogo intercultural permanente. En este sentido, las habilidades difieren de acuerdo con las particularidades y necesidades de cada organización y la cultura a la que pertenezca. Hoffman, Shipper, Davy y Rotondo (2012) reconocen en la dimensión cultural situaciones que inciden en los comportamientos, ya que configuran en los lugares de trabajo valores y creencias que repercuten en el direccionamiento estratégico organizacional. Por tanto, la alta gerencia habrá de dimensionar este tipo de situaciones y potenciar las habilidades directivas para convertirlas en ventajas competitivas.

\section{La dinámica de la clasificación de las habilidades directivas: consensos y divergencias}

Los estudios académicos a nivel global han intentado clasificar de forma objetiva la variedad de habilidades con las que debe contar la alta gerencia o los directivos de las organizaciones; sin embargo, se han encontrado con dificultades que estriban en la 
conceptualización delimitada de las habilidades puesto que, no sólo se trata de los conocimientos técnicos, también incorporan otros elementos sociales, políticos, éticos, entre otros que impactan directamente en las organizaciones a nivel interno y externo (Castellanos et al., 2019). Por otro lado, se establece que las condiciones y los contextos particulares de las organizaciones y donde ellas se circunscriben revelan la necesidad de establecer algunas habilidades y desestimar otras para evitar un detrimento en la organización. Es por esto que, a pesar de que se han creado grandes grupos conceptuales, cada uno integra una gran variedad de habilidades que se van constituyendo empíricamente (Cetina, Ortega y Aguilar, 2010).

En un primer momento se establece una clasificación general aceptada desde la década de 1980 en donde se estipulan tres grandes conjuntos de habilidades directivas: las habilidades técnicas u operativas, las habilidades conceptuales o administrativas y las habilidades sociales e interpersonales (Aburto y Bonales, 2011; Beenen y Pichler, 2016); en algunos casos el último grupo se divide puesto que se consideran que las habilidades interpersonales abarcan gran cantidad de elementos que se diferencian de las sociales en aspectos estructurales como las que conciernen al desarrollo psicosocial y las que se generan en la interacción con los grupos sociales. Las habilidades también se pueden clasificar como blandas o duras. En el primer grupo se establecen las sociales e interpersonales, y en el Segundo, las operativas y conceptuales. Esta última clasificación intenta concentrar una diferenciación clara que proviene desde los debates científicos por las disciplinas blandas y duras, esto con el fin de esclarecer en la literatura administrativa los aspectos clave de cada dimensión.

La habilidad operativa exige que los gerentes racionalicen las tecnologías, los recursos y las herramientas fundamentales para cumplir con los logros organizaciones de manera eficiente, aumentando la rentabilidad y las ventajas competitivas; se resaltan valores como la unidad, la integración y prácticas efectivas en la gestión de procesos y de rendimiento (Chen, Podolski, Veeraraghavan, 2015). Esta habilidad requiere la implementación y alineación de procesos claves, servicios de calidad y optimización en entornos empresariales que se prevean como turbulentos. Igualmente, se contemplan como inherentes a la formación de los administradores de empresas y de negocios, puesto que forma parte de la mayoría de los currículos alrededor del mundo; entre las más comunes en la literatura se observan: la comprensión de las áreas funcionales de un sistema, intercambio de información, operacionalización de las actividades, capacidad de optimización, conocimientos profundos del sector donde se desenvuelve, capacidad de gestión de procesos industriales, planificación, control de detalles y coordinación (Hoffman et al., 2012)

Las habilidades conceptuales se vislumbran en los conocimientos que tienen los gerentes y directivos en el momento de establecer una organización efectiva de las actividades 
colectivas. De acuerdo con Khan (2019) se destaca la capacidad de gestionar el conocimiento en las prácticas interactivas al interior de las organizaciones, se trata entonces, de implementar elementos pedagógicos continuos para la transmisión de las ideas, planes, objetivos, procesos y sistematización de todo lo que concierne a estas. Las habilidades conceptuales traen como resultado que los gerentes divulguen y transfieran otra habilidades pertinentes que posibiliten la coordinación, la optimización y el aumento de la capacidad organizacional con un colectivo cualificado. Adicionalmente, se establece que las habilidades conceptuales son todos aquellos conocimientos propios de la disciplina administrativa que el directivo puede poner en práctica de manera asertiva, demostrando su capacidad de resolución de problemáticas y de toma de decisiones racionales; además de cualidades analíticas, capacidad diagnóstica e integradora. Algunas de las más destacadas en la literatura son: conocimiento del mercado, resolución de problemas administrativos, superación de las amenazas del entorno, desarrollo de las fortalezas organizativas, gestión de la adversidad y establecer interrelaciones complejas entre lo económico y lo político asociado al funcionamiento de la organización.

En cuanto a las habilidades sociales e interpersonales, se pueden clasificar en: cognitivas, emocionales y sociales; en general se establece en la literatura que estas habilidades están direccionadas a mejorar el ambiente laboral, el clima organizacional y a resaltar valores éticos que contribuyen a la imagen que proyecta en el entorno (Cetina, Ortega y Aguilar, 2010; Codina, 2016) Las habilidades interpersonales más importantes consisten en la comunicación, control de conflictos, comprensión amplia de los comportamientos humanos, cohesión, entre otras. De acuerdo con Parente, Stephan y Brown (2012) son las más diversas y difíciles de identificar puesto que se remiten a elementos intangibles que no se pueden medir objetivamente. Sin embargo, revisten una gran importancia ya que sin estas, no sería posible bajo ninguna circunstancia gestionar el equipo de trabajo para lograr resultados dentro de los intereses comunes.

Las habilidades sociales trascurren en la vida cultural y social en la que está inmersa la organización, existen valores sociales propios de las culturales que se involucran en las capacidades de los gerentes, tanto como el sector económico en que se desenvuelven. Estas habilidades se remiten al campo de la subjetividad, de la particularidad y de la utilidad que se prevea para la organización. Si bien se trata de valores y capacidades en el campo de las relaciones, también se asocian a temas como la creatividad y las capacidades artísticas y estéticas, por tanto, existen claras diferenciaciones que debe establecer cada gerente con respecto a las habilidades que debe construir en el marco de su propia experiencia. En definitiva, es el campo más flexible de las habilidades y sólo pueden evidenciarse su valor en las situaciones colectivas cotidianas.

El afrontamiento de las crisis se considera una habilidad que converge entre las habilidades conceptuales, operativas y sociales (Codina, 2016). Puesto que la crisis no es un suceso 
definido, puede derivarse de muchas acciones dentro y fuera de la organización que si bien pueden ser previsibles no pueden ser solucionadas inmediatamente (Whetten y Cameron, 2011). Por tanto, se requiere de un profesional capacitado que pueda liderar estrategias de afrontamiento asertivas para evitar consecuencias a mediano y largo plazo (Tomastika, Strohmandlb y Cechc, 2015).

Tonidandel, Braddy y Fleenor (2012) adicionan otra dimensión de las habilidades sociales que si bien son constitutivas en los valores sociales, se remite también a la particularidad en el desempeño gerencial. Esta dimensión defiende el comportamiento ciudadano como un elemento clave de socialización con el entorno. Algunas de las habilidades requeridas para ello son la cooperación, lealtad, persistencia (Beenen y Pichler, 2016)

En la actualidad, las habilidades directivas no se delimitan a grupos definidos puesto que son necesarias en todos los ámbitos de la actividad gerencial. Para lograr cambios e innovaciones exitosas se requiere que los gerentes implementen habilidades técnicas, conceptuales e interpersonales simultáneamente; ejemplo de ello, es la necesidad de incorporar habilidades como la autogestión, la gestión de relaciones y las estrategias técnicas en un mismo objetivo organizacional para alcanzar los resultados esperados (Whetten y Cameron, 2011). Asimismo, se ha vislumbrado que en estudios sobre liderazgo existen habilidades específicas desarrolladas y sofisticadas que determinan el éxito de la gerencia como la inteligencia, capacidad estratégica, creatividad, diplomacia, fluidez, cohesión, capacidad administrativa, y fortalecimiento de las relaciones y valores sociales (Cetina, Ortega y Aguilar, 2010).

\section{Conclusiones}

El liderazgo es, por muchos autores, la habilidad más importante que interfiere en la toma de decisiones, en la consolidación de las relaciones interpersonales, y transversaliza todas las habilidades, puesto que depende de la capacidad de interacción con el medio, la efectividad y el éxito de las estrategias para las organizaciones (Cetina, Ortega y Aguilar, 2010; Codina, 2016; Villar y Araya, 2019). De acuerdo con Taucean, Tamasila y NegruStrauti (2016) a este concepto se le han atribuido una serie de conceptualizaciones orientadas al poder y a los medios de relacionamiento; en este sentido, se establece que existen dos tipos de liderazgos: el autocrático y el democrático, y ambos se desarrollan dependiendo de las interacciones interpersonales. Si estas están direccionadas a la apertura en la participación y en el diálogo continuo, entonces se trata de un liderazgo democrático, mientras que si se trata de un liderazgo en donde el director toma las decisiones unilateralmente, es de tipo autocrático (Villar, y Araya, 2019). Existen otros liderazgos como el paternalista, delegativo, el de gestión empobrecida, centrado en las relaciones interpersonales que afectan la gestión, autoritario con métodos de castigo y coerción, 
equilibrado con rendimientos promedio, y de compromiso bilateral con altos resultados. Este se considera el liderazgo ideal puesto que la gestión tiene dos preocupaciones: por las personas que conforman la organización y por la producción (Whetten y Cameron, 2011).

Por otra parte, la comunicación es el eje de las habilidades interpersonales (Whetten y Cameron, 2011; Codina, 2016). Giudici y Filimonau (2019) exponen que es un determinante en el liderazgo gerencial para las relaciones comunitarias dentro y fuera de las organizaciones. Es una de las habilidades que se pueden considerar escasas (Zat'ková y Poláček, 2015 ) teniendo en cuenta que la investigación empírica internacional ha definido que dentro de las organizaciones existen habilitadores e inhibidores que influyen en la interacción. Es una competencia interpersonal esencial que proyecta un efecto definitivo en la eficacia de las actuaciones de cada uno de los empleados (colaboradores) y en los stakeholders (Matemilola, Bany-Ariffin, Azman-Saini, 2013). Una comunicación ideal es la de tipo dinámica que se basa en la transmisión de mensajes verbales y no verbales decodificados concretos y efectivos para las labores rutinarias y extraordinarias de las organizaciones (Peña, 2005).

Finalmente, la toma de decisiones es una habilidad que consiste en la coordinación, delegación y centralización de las acciones que se desarrollan dentro de las organizaciones. Es una habilidad asociada a la productividad y a la consolidación de las ventajas competitivas; a su vez, integra la motivación (Whetten y Cameron, 2011), constitución de beneficios, incremento del valor social de los empleados y evita los conflictos en los procesos de cambio llevando al éxito organizacional (Codina, 2016)

\section{Referencias}

Aburto, H., Bonales, J. (2011). Habilidades directivas: Determinantes en el clima organizacional. Investigación y Ciencia, 19 (51), 41-49.

Ali, Z., Zwetsloot, I., Nada, N. (2019). Operational capabilities to infuse organizational innovation in SMEs. Procedia Computer Science, 158, 260-269.

Beenen, G., Pichler, S. (2016). A discussion forum on managerial interpersonal skills. Journal of Management Development, 35 (5), 706-716.

Bel, R., Smirnov, V., Wait, A. (2018). Managing change: Communication, managerial style and change in organizations. Economic Modelling, 69, 1-12.

Castellanos, J., Valle, F., Machado, A., García, N. (2019). Consideraciones metodológicas sobre la determinación de competencias directivas como factores claves del éxito empresarial. Centro Azúcar, 46 (4), 1-9. 
Cetina, T., Ortega, I., Aguilar., C. (2010). Habilidades directivas desde la percepción de los subordinados: Un enfoque relacional para el estudio del liderazgo. Psicoperspectivas, 9 (1), 124137.

Chen, J., Liu, X., Song, W., Zhou, S. (2020). General managerial skills and corporate social responsibility. Journal of Empirical Finance, 55, 43-59.

Chen, Y., Podolski, E., Veeraraghavan, M. (2015). Does managerial ability facilitate corporate innovative success? Journal of Empirical Finance, 34, 313-326.

Codina, A. (2016). Habilidades directivas. España: CITMATEL (IBD).

Giudici, M., Filimonau, V. (2019). Exploring the linkages between managerial leadership, communication and teamwork in successful event delivery. Tourism Management Perspectives, 32 .

Hoffman, R., Shipper, F., Davy, J., Rotondo, D. (2014). A cross-cultural study of managerial skills and effectiveness New insights or back to basics? International Journal of Organizational Analysis, 22 (3), 372-398.

Khan, M. (2019). Knowledge, skills and organizational capabilities for structural transformation. Structural Change and Economic Dynamics, 48, 42-52.

Matemilola, B., Bany-Ariffin, A., Azman-Saini, W. (2013). Impact of Leverage and Managerial Skills on Shareholders' Return. Procedia Economics and Finance, 7, $103-115$.

Parente, D., Stephan, J., Brown, R. (2012). Facilitating the acquisition of strategic skills. The role of traditional and soft managerial skills. Management Research Review, 35 (11), 1004-1028.

Peña, B.(2005). La dirección de comunicación y las habilidades directivas. Revista de Comunicación de la SEECI, (12), 36-54.

Puchol, L., Martín, M., Nuñez, A., Ongallo, C., Puchol, I., Sánchez, G. (2010). El libro de las habilidades directivas. México: Ediciones Díaz de Santos

Taucean, I., Tamasilaa, M., Negru-Strautia, G. (2016). Study on Management Styles and Managerial Power Types for a Large Organization. Procedia - Social and Behavioral Sciences, 221, 66 - 75.

Tomastika, M., Strohmandlb, J., Cechc, P. (2015). Managerial competency of crisis managers. Procedia - Social and Behavioral Sciences, 174, 3964 - 3969.

Tonidandel, S., Braddy, P., Fleenor, J. (2012). Relative importance of managerial skills for predicting effectiveness. Journal of Managerial Psychology, 27 (6), 636-655.

Toro, I., Parra, R. (2010) Fundamentos epistemológicos de la investigación y la metodología de la investigación cualitativa/cuantitativa. Medellín: Editorial Universidad EAFIT.

Varela, O., Burke, M., Michel, N. (2013). The development of managerial skills in MBA programs. A reconsideration of learning goals and assessment procedures. Journal of Management Development, 32 (4), 435-452.

Villar, M., Araya, L. (2019). Consistencia entre el enfoque de liderazgo y los estilos de liderar: clave para la transformación y el cambio. Pensamiento \& gestión, 46, 187-221. 
Whetten, D., Cameron, K. (2011). Desarrollo de habilidades directivas. México: Pearson Educación.

Zat'ková, T., Poláček, M. (2015). Social Skills as an Important Pillar of Managerial Success. Procedia Economics and Finance, 34, 587 - 593. 